\title{
Survival after Esophageal Rupture during the COVID Pandemic: The Evolution of a Mallory Weiss Tear to Boerhaave's Syndrome
}

\author{
Kaczynski $\mathrm{RE}^{1^{*}}$, Rogers $\mathrm{EH}^{1}$, Amador-Jimenez $\mathrm{J}^{2}$, Shakir $\mathrm{H}^{3}$, Colaco $\mathrm{R}^{4}$, Battista ${ }^{1}$ \\ ${ }^{1}$ Department of Surgery, St. John's Episcopal Hospital, Far Rockaway, USA \\ ${ }^{2}$ St. Georges University School of Medicine, West Indies, Grenada \\ ${ }^{3}$ Assistant Professor of Surgery, Division of Cardiothoracic Surgery, Rutgers New Jersey Medical School, Newark, New Jersey, \\ USA \\ ${ }^{4}$ Department of Surgery, Trinitas Regional Medical Center, Elizabeth, New Jersey, USA
}

Corresponding Author: Rachel E. Kaczynski

Address: Department of Surgery, St. John's Episcopal Hospital, Far Rockaway, New York 11691, USA.

Received date: 10 December 2020; Accepted date: 02 January 2021; Published date: 09 January 2021

Citation: Kaczynski RE, Rogers EH, Amador-Jimenez J, Shakir H, Colaco R, Battista J. Survival after Esophageal Rupture during the COVID Pandemic: The Evolution of a Mallory Weiss Tear to Boerhaave's Syndrome. Asp Biomed Clin Case Rep. 2021 Jan 09;4(1):22-29.

Copyright (C) 2021 Kaczynski RE, Rogers EH, Amador-Jimenez J, Shakir H, Colaco R, Battista J. This is an openaccess article distributed under the Creative Commons Attribution License, which permits unrestricted use, distribution, and reproduction in any medium provided the original work is properly cited.

\section{Keywords}

Boerhaave Syndrome, Mallory-Weiss Tear, Distal Esophageal Rupture, COVID-19, Pandemic

\section{Introduction}

We present the case of a 42-year-old male cirrhotic chronic alcoholic who was admitted during the height of the COVID pandemic with a large right pleural effusion. Thorough investigation revealed a large right-sided distal esophageal rupture near the gastroesophageal junction and he was diagnosed with Mallory Weiss tear converted to Boerhaave's syndrome. He successfully underwent endoscopic placement of a covered esophageal stent, but had a protracted recovery with presumed empyema continuing to require chest tube drainage. He eventually required surgical intervention with a right thoracotomy, decortication, and wash out. Our case provides an excellent example of the risk of distraction during a global pandemic secondary to nonspecific symptomatology being attributed to COVID-19 and significant critical care requirements leading to a significant delay in diagnosis of an esophageal rupture. However, our patient is also uniquely impressive when compared to similarly published cases of Mallory Weiss conversion to Boerhaave's Syndrome given his survival with excellent clinical outcome leading to discharge home on oral diet despite his increased risk of morbidity based on his prolonged critical illness disease course.

\section{Case Report}

We present the case of a 42 -year-old male with a history of alcohol and cocaine abuse who was brought to the emergency department from home in April 2020 at the height of the COVID-19 pandemic in the Greater New York City area. As per his family, he complained of hematemesis leading to shortness of breath, and eventually, he became unresponsive. He was admitted to the ICU as a person under investigation for COVID19. Gastroenterology was consulted, but endoscopy was deferred given the patient's clinically tenuous status and lack of overt GI bleeding. Our patient's respiratory status continued to deteriorate and a chest $\mathrm{x}$-ray demonstrated a large right pleural effusion 
Citation: Kaczynski RE, Rogers EH, Amador-Jimenez J, Shakir H, Colaco R, Battista J. Survival after Esophageal Rupture during the COVID Pandemic: The Evolution of a Mallory Weiss Tear to Boerhaave's Syndrome. Asp Biomed Clin Case Rep. 2021 Jan 09;4(1):22-29.

\section{Case Report}

prompting general surgery consultation for chest tube placement. Upon evaluation by the surgical team, the patient appeared toxic and was in respiratory distress. He was saturating $90-92 \%$ on $15 \mathrm{~L}$ oxygen via nonrebreather, tachypneic to 40 breaths per minute, using accessory muscles of respiration, and breath sounds on the right were severely decreased. Additionally, on physical examination, he had a distended abdomen with fluid wave consistent with ascites. Laboratory examination demonstrated Child-Pugh Class B cirrhosis, leukocytosis of 20,000 white blood cells $/ \mathrm{mm}^{3}$, and acute versus chronic kidney injury with a creatinine of $2.3 \mathrm{mg} / \mathrm{dL}$. A $14 \mathrm{~F}$ pigtail catheter was placed in the right fifth intercostal space in the anterior axillary line initially yielding 2 liters of dirty dishwater colored fluid.

Over the following days, our patient improved clinically, was found to be COVID-19 negative, and was downgraded to the medical floor. However, he later became febrile and tachycardic but with decreasing oxygen requirements. Non-contrast computed tomography (CT) of his chest demonstrated a persistent right-sided pleural effusion containing multi-loculated fluid collections with air-fluid levels around the thoracostomy tube. Cardiothoracic surgery was consulted and determined that the patient was not a surgical candidate as his clinical status would not tolerate single lung intubation or surgical stress. The pigtail thoracostomy tube was continued to a chest drainage system on suction and antibiotics were continued with a regimen tailored to pleural cultures by an infectious disease which eventually speciated to Candida Albicans, which was also observed in the patient's sputum. Our patient later returned to the ICU on an Ativan drip due to alcohol withdrawal which had progressed to delirium tremens. His thoracostomy tube remained on suction but subsequent chest $\mathrm{x}$-rays did not show improvement of the effusion despite changes in antibiotic regimen including antifungal coverage. He underwent interventional radiology placement of a peripherally inserted central venous catheter (PICC) for total parental nutrition (TPN), and two new image guided pigtail thoracostomy tubes into the loculations, and the prior thoracostomy tube was removed (Fig-1). The pigtail catheters initially drained $300 \mathrm{~mL}$ of purulent fluid superiorly, and $50 \mathrm{~mL}$ of purulent drainage inferiorly.

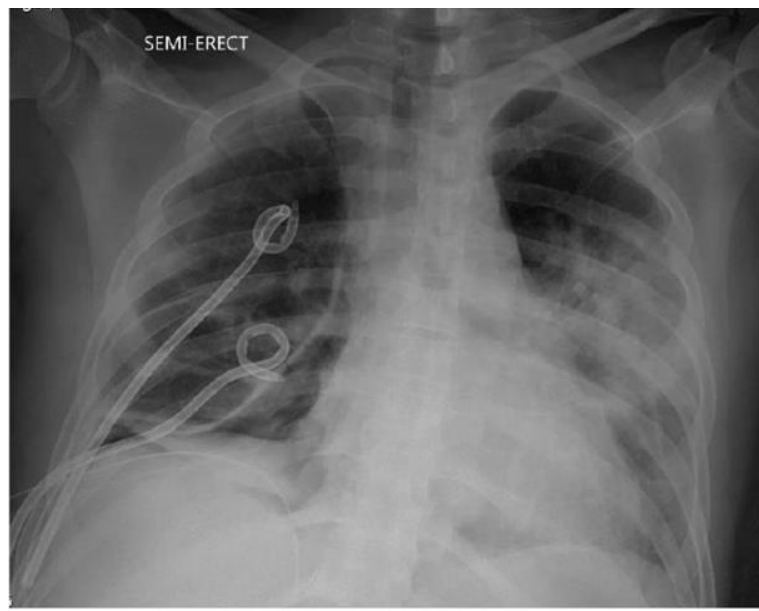

Fig-1:

Anterior-Posterior (AP) chest $x$-ray demonstrating two $14 F$ pigtail thoracostomy tubes in right hemithorax.

After aggressive medical management, antibiotics, and recovery from alcohol withdrawal, our patient's thoracostomy tube outputs had decreased; however, he had persistent effusion on imaging prompting upsizing of the pigtail tubes to $28 \mathrm{~F}$ chest tubes to allow more adequate drainage (Fig-2). On hospital day 17, his medical conditions and mental status had improved enough for the medical team to begin enteral nutrition with a clear liquid diet. Several days later, he was observed to have a bright green output in his chest tube drainage system after eating green Jell-O. Upon re-evaluation by Cardiothoracic surgery,

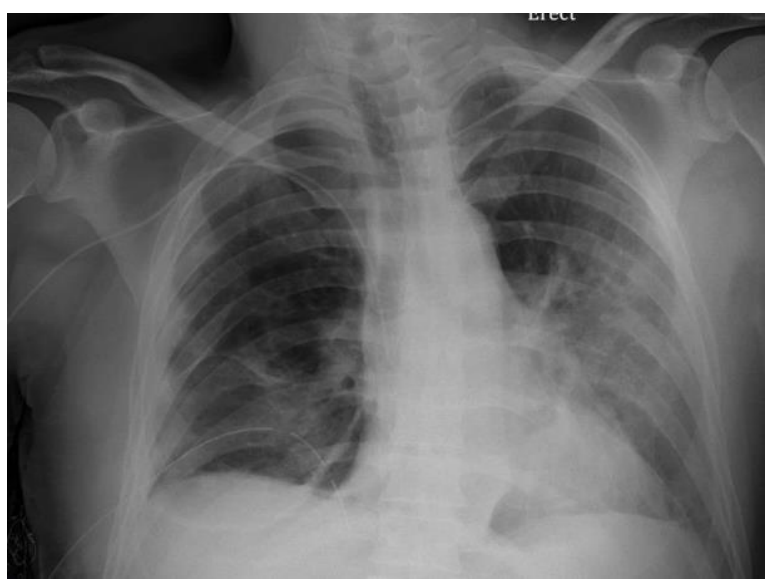

Fig-2:

$A P$ chest $x$-ray demonstrating upsizing to two $28 F$ thoracostomy tubes in right hemithorax. 
Citation: Kaczynski RE, Rogers EH, Amador-Jimenez J, Shakir H, Colaco R, Battista J. Survival after Esophageal Rupture during the COVID Pandemic: The Evolution of a Mallory Weiss Tear to Boerhaave's Syndrome. Asp Biomed Clin Case Rep. 2021 Jan 09;4(1):22-29.

\section{Case Report}

his chest tube output was noted to be clear and foamy rather than purulent or straw-colored and raised concern for salivary contents as opposed to a traditional transudative effusion. At this time our patient was also able to provide further history as he now recalled events leading up to his hospitalization. Despite forceful retching, he admitted to the continuation of beer and red wine consumption and intranasal use of cocaine over the course of three days. He reported his final memory prior to this day was that of severe shortness of breath and blacking out in his bedroom with his spouse attempting to revive him but he was unable to respond.

At this time, our patient's vitals had normalized. He required minimal to no supplemental oxygen and had non-labored respirations on room air when examined by the Cardiothoracic surgery team. Differential diagnosis included chronic aspiration with empyema and lung abscess secondary to chronic alcohol intoxication, or an esophageal-pleural fistula secondary to esophageal disruption from forceful retching. A gastrografin swallow evaluation demonstrated a distal esophageal perforation with the preferential flow of contrast into the right pleural cavity (Fig-3).

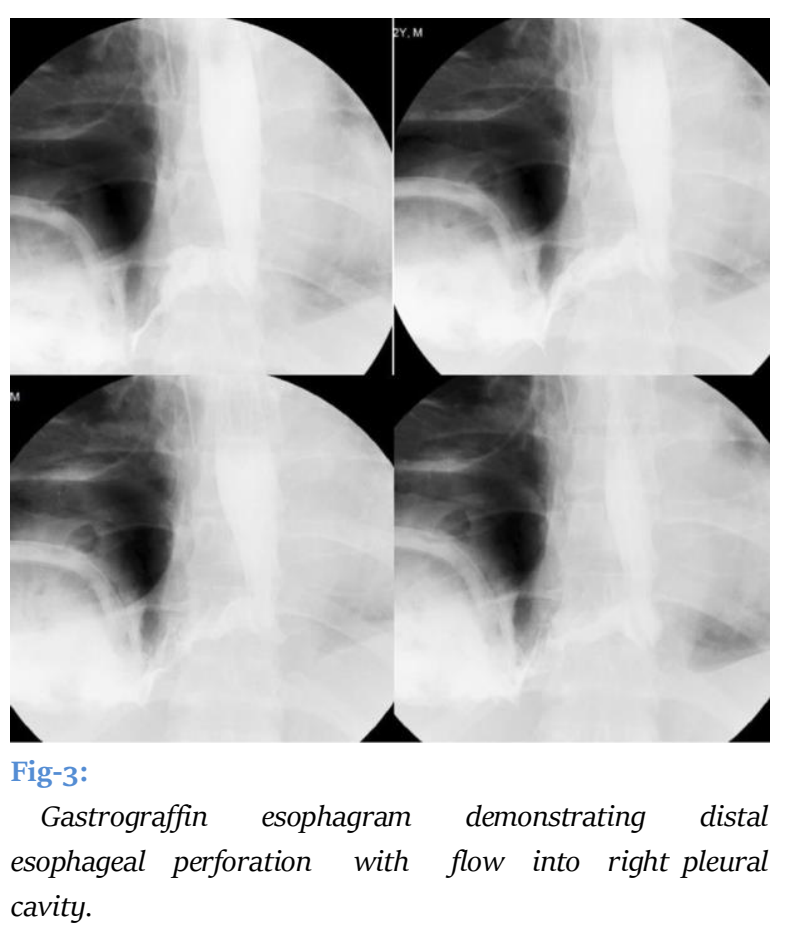

Potential treatment options were explained and our patient consented to a proposed cervical spit fistula and feeding jejunostomy tube placement. $\mathrm{He}$ understood that he may also require thoracotomy and decortication for empyema if attempting to control his infection and effusion with the chest tubes was unsuccessful postoperatively. However, after extensive discussions between the general surgery, Cardiothoracic surgery, and gastroenterology teams, it was decided to first attempt less invasive management with endoscopic covered stent placement over the perforation and percutaneous endoscopic gastrostomy (PEG) tube placement by gastroenterology. After this procedure was completed, the medical team began PEG tube feedings which caused an immediate increase in chest tube output, prompting conversion to a feeding jejunostomy tube with the hopes of decreasing pressure from feedings on the area of perforation given its proximity to the gastroesophageal junction.

A repeat esophagram was performed demonstrating a small amount of contrast leak external to the esophageal stent into the right pleural space and a small amount of esophageal reflux above the level of the stent. CT of the chest with oral contrast demonstrated improvement of the empyema with a small persistent effusion and loculated pocket of air. Jejunostomy tube feedings at that time were also associated with increased chest tube output, concerning for continued esophageal leak. Repeat fluid analysis at this time revealed fluid with a $\mathrm{pH}$ of 7.5 , amylase of $18,477 \mathrm{u} / \mathrm{L}$, total protein of $3.7 \mathrm{~g} / \mathrm{dL}$, red blood cell count of 569,250 cells $/ \mathrm{mm}^{3}$, and white blood cell count of 85,770 cells $/ \mathrm{mm}^{3}$ with a differential of $94 \%$ neutrophils. Fluid lipid analysis and chylomicrons were unable to be obtained due to laboratory capabilities. Cultures demonstrated moderate coagulase negative Staphylococcus and Stenotrophomonas maltophilia, and Klebsiella oxytocci. Cardiothoracic surgery team recommended a venting gastrostomy tube to decrease pressure on the gastroesophageal junction and distal esophagus from swallowed oral contents and refluxing jejunostomy feeds, which was placed by 
Citation: Kaczynski RE, Rogers EH, Amador-Jimenez J, Shakir H, Colaco R, Battista J. Survival after Esophageal Rupture during the COVID Pandemic: The Evolution of a Mallory Weiss Tear to Boerhaave's Syndrome. Asp Biomed Clin Case Rep. 2021 Jan 09;4(1):22-29.

Case Report

gastroenterology several days later.

Our patient continued to improve and was advanced to clear liquid diet which he tolerated well with intermittent requirement for venting from the gastrostomy tube. A repeat chest CT demonstrated loculated collection of air regional to the chest tube within lower right hemithorax with radiodense structures in the dependent aspect of this space. A repeat gastrografin esophagram demonstrated no leak or aspiration. Chest tube output remained minimal and our patient continued to tolerate clear liquid diet; therefore, supplemental jejunostomy tube feedings were stopped. On hospital day 54, the remaining chest tube was removed which the patient tolerated well. Chest CT with oral contrast the following day demonstrated a very small leak of contrast between the esophageal wall and the stent at the site of previous perforation and a small area of round atelectasis in the right hemithorax anterior to a small amount of pleural fluid. Cardiothoracic surgery recommended that the patient returns to NPO status with TPN for 3 to 4 weeks to allow for full healing of the perforation. However, he was discharged by the medical team several days later with instructions to advance diet as tolerated.

After one week at home on a regular diet, our patient returned to the hospital complaining of burning post-prandial chest pain. Laboratory evaluation revealed a leukocytosis of 17,500 cells $/ \mathrm{mm}^{3}$, and imaging demonstrated a right pleural effusion with a $10.8 \mathrm{~cm}$ by $6.2 \mathrm{~cm}$ area of loculated air and fluid collection in the posterior right hemithorax. Gastrografin esophagram demonstrated no evidence of an esophageal leak, and interventional radiology was consulted for drainage which they deferred as the loculated effusion was unlikely to be amenable to percutaneous drainage alone. Cardiothoracic surgery was consulted and consented our patient for right chest video assisted thoracoscopy (VATS) and possible open thoracotomy with decortication for chronic empyema due to chronic effusion of gastric contents. He was brought to the operating room where VATS was attempted but quickly converted to open thoracotomy due to poor visualization secondary to dense adhesions in the pleural space. During the open thoracotomy, a single rib resection was required to obtain adequate visualization and room for adhesiolysis. Two large areas of loculated effusions were discovered with gross purulence in the posterior inferior loculation. This purulent material was suctioned and sent for a culture which grew Klebsiella oxytoca, Enterococcus faecalis group D, and Candida albicans. A $0.5 \mathrm{~cm}$ calcified object was removed from this cavity along with three metallic clips from previously attempted endoscopic management (Fig-4). Two large bore chest tubes were placed into each loculated pocket, the fascia was closed, and the skin was closed with widely spaced interrupted skin closure with wound vac placement to prevent post-operative soft tissue infection. Final pathology reports demonstrated an area of acute osteomyelitis in the resected rib, amorphous calcific material with scattered neutrophils and macrophages, and metallic foreign bodies thought to be esophageal clips from previous endoscopic treatment of the esophageal tear.

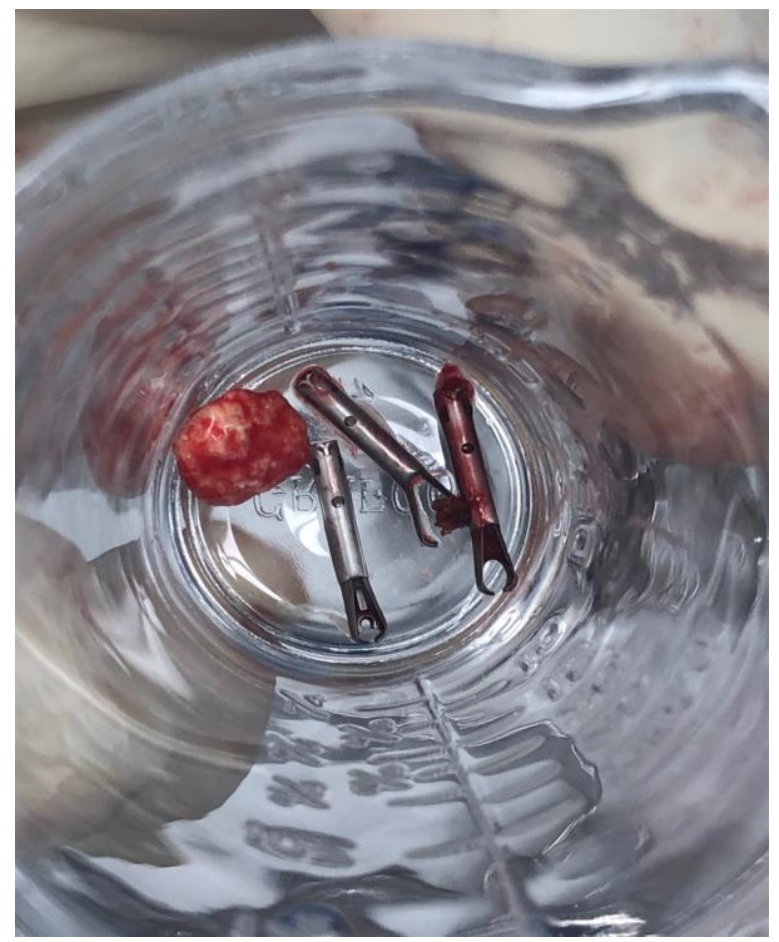

Fig-4:

Calcified material and three metallic endoscopic clips removed from right hemithorax during thoracotomy. 
His postoperative course was complicated with the development of acute kidney injury which was managed with intravenous fluid hydration by nephrology. Our patient was transitioned from parenteral nutrition to enteral feeding via jejunostomy tube with the gastrostomy tube clamped. At this time, he developed nausea and persistent emesis with any rate of feeding over $10 \mathrm{~mL}$ per hour. The gastrostomy tube was replaced to venting suction and jejunostomy tube feedings were held. The chest tube output was also noted to be thick and milky white in consistency, raising concern for chylothorax after initiation of a diet containing lipids. Our patient was slowly advanced to clear liquid diet with TPN supplementation without intralipids. His chest tube output cleared up over the next several days and he continued to improve clinically. The chest tubes and wound vac were removed according to patient progression and his labs normalized. He was discharged home on low fat, medium chain triglyceride clear liquid diet and has subsequently been advanced to a more substantial diet without complications. In the months following, our patient has required another admission for pigtail thoracostomy drainage of pleural fluid collections and endoscopic re-positioning of the stent, but he has recovered well and is tolerating a regular diet without restrictions.

\section{Discussion}

Spontaneous esophageal rupture (Boerhaave's syndrome) can manifest as an array of symptoms including classic Mackler's triad of vomiting, lower thoracic pain, and subcutaneous emphysema [1,2], as well as less common symptoms including dysphagia, tachycardia, fever, tachypnea, and epigastric pain [3]. These perforations are usually longitudinal, average $22 \mathrm{~mm}$ in length, and are most common approximately $3-4 \mathrm{~cm}$ above the gastroesophageal junction [1-3]. Furthermore, esophageal rupture is most common on the left side due to anatomical weakness of the left posterolateral aspect of the esophagus just above the diaphragm, resulting in fullthickness rupture of the esophagus, whereas MalloryWeiss tears are more likely to occur on the right side of the esophagus [1-3]. It is thought that only $50 \%$ of Boerhaave's syndrome present with Mackler's triad which contributes to a significant delay in diagnosis for many patients. The historical treatment pathway has been centered on a prompt diagnosis with urgent thoracic drainage and damage control surgery [1]. Other cases have documented delayed presentation due to initial misdiagnosis and recommend that Boerhaave's syndrome be considered in all ill-appearing patients presenting with a combination of gastrointestinal and respiratory complaints [4]. Principles of management are the same for all patients regardless of comorbidities, including sepsis control by limiting diffuse contamination, adequate drainage, perforation repair, and antibiotic therapy to prevent death from sepsis and multiple organ failure $[1,4]$.

Currently, only three publications report conversion of Mallory-Weiss tear to Boerhaave's syndrome. McVay et al. first reported a case of conversion in 1970, and no further reports were made of this phenomenon until 2002 when Marshall documented a case from the anesthesia perspective after 15 days of management in the intensive care unit $[5,6]$. In 2018, Cucci et al. reported a case of a 45-year-old male who unfortunately succumbed to cardiorespiratory arrest attributed to pleural effusion after spontaneous transition from MalloryWeiss lesion to Boerhaave's syndrome over several days. Diagnosis of Boerhaave's syndrome greater than 24 hours from the onset of symptoms has been proven to increase risk for fatal outcome $[3,7]$.

A retrospective review of 34 patients with spontaneous esophageal rupture over 30 years found that $50 \%$ of patients had a delay in diagnosis defined as the time from onset of symptoms to establishment of correct diagnosis exceeding 24 hours. These delays were often secondary to delay in patient presentation and incorrect initial diagnosis, with $77 \%$ resulting from diagnostic delay as the most common cause. This review determined that the delay in diagnosis did not affect mortality rates, with a mortality of $33 \%$ in those patients diagnosed within the first 24 hours, and $34 \%$ in those diagnosed after 24 hours. They stressed the keys to successful management based on their data were broad-spectrum antibiotics for treatment 
of mediastinitis, nasogastric decompression, sump drainage of the upper esophagus, antibiotic fluids by mouth, correction of fluid and electrolytes as well as blood volumes before during, and after any operative intervention [8]. Five years later, Troum et al. reported the first published case of surviving Boerhaave's syndrome with free pleural rupture without requiring thoracotomy in 1994 [9]. Later, Jagminas et al. (1996), reported a case of delayed diagnosis of Boerhaave's syndrome with right-sided esophageal perforation due to unusual symptoms on initial presentation [4].

More recently, information has been published detailing non-surgical management of Boerhaave's syndrome with a two-year follow-up [2]. There were no deaths in the study group, and clinical resolution was achieved in all patients without surgical intervention. However, the mortality rate observed for patients with a delay in diagnosis in this small case series was $30-50 \%$. They propose that the delay in diagnosis of Boerhaave's diagnosis is due to the vague clinical picture when compared to iatrogenic rupture, thus leading to increased risk of mortality. The most common site for perforation was the distal esophagus, $3 \mathrm{~cm}$ above the gastroesophageal junction. $83.3 \%$ of patients required mechanical ventilation for respiratory failure. $100 \%$ of the patients had endoscopic treatment with fully covered esophageal stents and had interventional radiology guided drainage of mediastinal and pleural fluid. 50\% of these patients required repeat stenting due to stent migration or continuous leakage. $66.6 \%$ of the patients had subsequent feeding tube placement via gastrostomy or jejunostomy for enteral nutrition until complete resolution. They also report that Boerhaave's diagnosis is often delayed due to vague clinical picture when compared to iatrogenic rupture, thus leading to detrimental effects on survival. This recent information proposes that esophageal stenting is sufficient management of complete esophageal rupture to prevent continued septic contamination and guides re-epithelialization of esophageal mucosa with stent placement or endoscopic suture. The authors propose that closure of the mucosal defect also allows for early enteral feeding and a reduction in morbidity and mortality when compared to surgical management. However, the main drawbacks of esophageal stenting alone include migration, pressureinduced ischemia, ulceration, perforation, new reactive stenosis at the ends of the endo-prosthesis, bleeding or injury upon removal of the stent, and unsuccessful retrieval of device later [2]. In our case, we also discovered a risk of misplacement of endoscopic clips through the perforation into the chest creating a significant risk for morbidity that should not be overlooked in patients with ongoing empyema or pleural effusions due to foreign body reaction.

In the past, nonoperative management of esophageal perforations was only considered for patients with circumscribed transmural perforation, non-neoplastic tissue, perforations not extending into the abdominal cavity, or accompanied by simultaneous obstructive esophageal disease, and without evidence of septicemia [10]. A retrospective review of all treatment options for management of esophageal perforations in 44 patients over 12 years found that $27 \%$ of the patients underwent esophageal endoscopic stenting, $55 \%$ of the patients underwent surgical management, and 18\% underwent conservative treatment with the cessation of oral intake, antibiotics, and parenteral nutrition. Of the surgically managed patients, 20\% required suture closure of the lesion, 32\% required esophagectomy with delayed reconstruction, and $1 \%$ required distal esophageal resection with gastrectomy. Overall, this retrospective review observed that $89 \%$ of patients with Boerhaave's syndrome underwent surgical management [11]. More recently, Schweigert et al. compared outcomes for endoscopic stent placement versus primary operative management for Boerhaave's syndrome. Similar to our patient's case, they report $84.6 \%$ of patients who underwent endoscopic stenting developed either severe pleural empyema or sustained mediastinal abscess formation requiring operative management. The reported patients requiring operative intervention underwent VATS, thoracotomy, or mediastinotomy, and $23 \%$ of these patients required multiple surgical interventions. This study is unique because it only evaluated patients with spontaneous rupture due to 
Citation: Kaczynski RE, Rogers EH, Amador-Jimenez J, Shakir H, Colaco R, Battista J. Survival after Esophageal Rupture during the COVID Pandemic: The Evolution of a Mallory Weiss Tear to Boerhaave's Syndrome. Asp Biomed Clin Case Rep. 2021 Jan 09;4(1):22-29.

Case Report

Boerhaave's syndrome and eliminated data from iatrogenic esophageal perforations and those resulting from esophageal malignancy. Although the surgical intervention was necessary to manage infectious complications from gastric contents in the chest, mortality rates were not statistically significant between the stent group and surgical group [7].

\section{Conclusion}

Boerhaave's syndrome should be considered in all ill-appearing patients presenting with a combination of gastrointestinal and respiratory complaints [4]. Unfortunately, our patient's full history of the events leading up to his presentation were not obtained until several weeks later due to altered mental status and critical illness. Historically, nonoperative management of esophageal perforations was only considered for patients in specific situations [10]. However, more recently, it has been shown that a delay in diagnosis more than 24 hours after onset of symptoms in patients with Boerhaave's syndrome increases risk of fatal outcome overall but without statistically significant differences in mortality between patients treated with endoscopic stenting versus operative management $[2,7,8]$. Our patient presents an interesting discussion of diagnostic and management delays likely influenced by vague clinical presentation, resource depletion during the COVID-19 pandemic, and development of complicated medical conditions within his hospitalization. These complex factors make his discharge home on oral diet uniquely impressive when compared to similarly published cases of Mallory Weiss conversion to Boerhaave's syndrome.

\section{Disclosures}

We have consent from our patient for publication of his case in compliance with HIPAA.

\section{Conflict of Interests}

All authors have read and approved the final version of the manuscript. The authors have no conflicts of interest to declare.

\section{References}

[1] Catarino Santos S, Barbosa B, Sá M, Constantino J, Casimiro C. Boerhaave's syndrome: A case report of damage control approach. Int J Surg Case Rep. 2019;58:104-107. [PMID: 31029781]

[2] Aloreidi K, Patel B, Ridgway T, Yeager T, Atiq M. Non-surgical management of Boerhaave's syndrome: a case series study and review of the literature. Endosc Int Open. 2018 Jan;6(1):E92-E97. [PMID: 29344568]

[3] Cuccì M, Caputo F, Fraternali Orcioni G, Roncallo A, Ventura F. Transition of a Mallory-Weiss syndrome to a Boerhaave syndrome confirmed by anamnestic, necroscopic, and autopsy data: A case report. Medicine (Baltimore). 2018 Dec;97(49):e13191. [PMID: 30544378]

[4] Jagminas L, Silverman RA. Boerhaave's syndrome presenting with abdominal pain and right hydropneumothorax. Am J Emerg Med. 1996 Jan;14(1):53-56. [PMID: 8630158]

[5] McVay CB. Treatment of a Case of Combined Mallory-Weiss and Boerhaave's Syndrome. Surg Clin North Am. 1970;50(5):1111-17.

[6] Marshall WB. Boerhaave syndrome: a case report. AANA J. 2002 Aug;70(4):289-92. [PMID: 12242927]

[7] Schweigert M, Beattie R, Solymosi N, Booth K, Dubecz A, Muir A, Moskorz K, Stadlhuber RJ, Ofner D, McGuigan J, Stein HJ. Endoscopic stent insertion versus primary operative management for spontaneous rupture of the esophagus (Boerhaave syndrome): an international study comparing the outcome. Am Surg. 2013 Jun;79(6):634-40. [PMID: 23711276]

[8] Pate JW, Walker WA, Cole FH Jr, Owen EW, Johnson WH. Spontaneous rupture of the esophagus: a 30-year experience. Ann Thorac Surg. 1989 May;47(5):689-92. [PMID: 2730190]

[9] Troum S, Lane CE, Dalton ML Jr. Surviving Boerhaave's syndrome without thoracotomy. Chest. 1994 Jul;106(1):297-99. [PMID: 8020294]

[10] Altorjay A, Kiss J, Vörös A, Bohák A. Nonoperative management of esophageal perforations. Is it justified? Ann Surg. 1997 Apr;225(4):415-21. [PMID: 9114801]

[11] Vallböhmer D, Hölscher AH, Hölscher M, Bludau M, Gutschow C, Stippel D, Bollschweiler E, Schröder W. Options in the management of 
Citation: Kaczynski RE, Rogers EH, Amador-Jimenez J, Shakir H, Colaco R, Battista J. Survival after Esophageal Rupture during the COVID Pandemic: The Evolution of a Mallory Weiss Tear to Boerhaave's Syndrome. Asp Biomed Clin Case Rep. 2021 Jan 09;4(1):22-29.

\section{Case Report}

esophageal perforation: analysis over a 12-year period. Dis Esophagus. 2010 Apr;23(3):185-90. [PMID: $19863642]$ 